\title{
Processing of parts for aerospace engineering
}

\author{
Alexander I. Boldyrev ${ }^{1, *}$, Alexander A. Boldyrev ${ }^{1}$, and Oleg N. Fedonin ${ }^{2}$ \\ ${ }^{1}$ Voronezh State Technical University, 14, Moscow Avenue, 394026, Voronezh, Russia \\ ${ }^{2}$ Bryansk State Technical University, 7, 50-letiya Octyabrya Blvd, 394026, Bryansk, Russia
}

\begin{abstract}
The article is devoted to combined processing techniques applied in modern industrial production for fabrication of aerospace engineering parts. The attainable process parameters are found for each technique. It is shown that electrochemical mechanical processing technique has maximal technological capability, this allows the increase of endurance strength and decrease of item mass.
\end{abstract}

\section{Introduction}

The combination of mechanical impact with other kinds of impact may cause maximum effect and opens the new doors for broadening of industrial technological capability during the development of high technology competitive products. The technologies and means of electrochemical mechanical processing often provide the broadening of process capabilities and production of new and novel effect.

Techniques of electrochemical mechanical processing are relating to combined ones, such are processes where chemical impacts and mechanical impacts both direct and impulse ones are used, they have a great impact on the increase of operation factors of products. Electric field in this case is not only the source of energy but also intensifying factor that increases the effect of base impacts.

The worked out means and appliances caused the development of revolutionary control system for the processes of combined electrochemical mechanical processing which open doors to achievement of high accuracy, low roughness and ensure the accomplishment of fatigue strength limit that provides the supplies of long-lasting products performance in multicycle loadings that are open in particular for aerospace and other transport equipment. During scientific justification of guaranteed fatigue enhancement developers of current technology have an opportunity to lower the mass of load-bearing elements, this open doors to yield of competitive indusctial product output.

\section{Combined processing techniques}

The method of electroabrasive processing is widely applied in production of nozzle, rotating blades of gas turbines and compressors made of structural chromium steels (13Cr12NiWoMoWA, Cr17Ni2, 30CrMnSiA, etc.), aluminum (VK4, AK6, VL-17, etc.), titanium (VT3, VT4, VT9, VT3-1, etc.) and high-temperature alloys (CrNi17TiAlB,

\footnotetext{
* Corresponding author: alexboldyrev@,mail.ru.
} 
CrNi70MoWoAlNb, HTA3, HTA6-K, etc.) [2]. Such processing provides stable surface finish $=0,32-0,64$ micron. Besides, current imposition allows decrease of cycle discharge by $10-15$ times without loss of profile accuracy.

Electroabrasive profile finish grinding of thermal (quenching) and chemical thermal (casehardening) reinforced gear wheels provides profile accuracy within 0,003-0,004 mm, for spline joints $-0,008-0,01 \mathrm{~mm}$ with surface finish $R a=0,08-064$ micron [4]. The labour coefficient of part processing equals from 6 to 12 min with tool wear of about $0,1 \%$.

The technique is also effective in processing of hard conductive materials, in particular oxide-carbide mineral-ceramic and metal-ceramic alloys. Processing by diamond wheels at direct polarity increases the speed of material removal up to 3 times and lowers wheels wear up to 4 times. During combined finish by diamond wheels it is recommended to use the grain concentration up to $100 \%$, and fulfill processing in operating media with $5-9 \%$ contain $\mathrm{Na}_{3} \mathrm{PO}_{4}$ or $\mathrm{Na}_{2} \mathrm{HPO}_{4}$ 1,5-3\% $\mathrm{Na}_{2} \mathrm{CO}_{3}$ addition of urotropine and humectant [5].

Vibroabrasive electrochemical technique is used mainly for processing parts made of non-ferrous metals and alloys and also for parts made of ferrous metals and alloys with complex shape, screwed internal cavities, hard to process spots. Such combined processing is used in main branches of industry. But the most effectively it is applied in aviation and space branches for the production of turbines and impellers made of titanium and nickel alloys, set of nozzles, confusers, jets with high requirements concerning specific finish control and uniformity of coldworking depth. This technique is promising for pump parts and turbocharging aggregates of transport machines, for which such combined finishharden processing allows the increase of their endurance by $20-60 \%$ [6].

It is deduced from experiments that during vidroabrasive electrochemical processing of high-resistance steels (such as 30ХГСНА) it is possible to increase the amount of area efficiency by 50-100 times. After processing the surface has light appearance, the surface finish equals $R a=0,32-1,25$ micron. In this case there appears increase of corrosion and endurance strength by $2-2,5$ times [7].

Technique of processing in electrolyte with filler in industrial production is applied for nondimensional and dimensional local stock removal in hardly accessible by tool spots and fine workpieces, this provides defined indices of surface layer for the increase of service properties for the parts operating under alternate loads. This electrochemical mechanical processing is rather effective for local finishing and hardening of parts in gas turbine pump aggregates, because it increases their operational life by $15-20 \%$ [8].

Combined processing by nonprofile shape tool (brush electrode) allows replacement of labour-consuming draft operations of lap removal from melted centrifugal wheels made of heat resistant alloy workpieces, deburring, and rounding of sharp ridges and transition areas of blanked and machined blades, embedded items and tie bands made of heat-resistant and titanium alloys and achieve high finish accuracy and dimensional finishing for surfacing before electrolyte coatings.

In [9] it is shown that during draft operations of lap removal from cast grids made of grey cast iron (brush with bristles made of hard brass or steel wire) combined processing by nonprofile tool reduces processing time up to 7 times in comparison with machining ensuring imprecision up to $\pm 0,3 \mathrm{~mm}$ and surface finish $R a=5-6$ micron. Processing at finishing modes (brush with bristles made of soft brass wire) is still more effective. So in processing of molded cases, centrifugal wheels and turbine blades the achievable imprecision equals $\pm 0,2 \mathrm{~mm}$ with surface finish $R a=3,2-5$ micron, and the capability increases up to 4 times, because the process is performed during one operating cycle. Positive results are also achieved in processing of fine workpieces made of ferrous and nonferrous alloys. 


\section{Electrochemical mechanical processing technique}

Though well-known investigations in this field deal with achievement of one or two parameters and cannot cover the full range of designers' requirements. Application of combined processing techniques according to customary control circuits as a rule solve local problems. In 1980s engineers proposed a new method of processing control by amount of contact pressure [10], which provided warranted cold work, high accuracy, low roughness, and top performance during processing of uniform cross-section channels.

Some practices were made in connection with shaping of outer surfaces having different cross-sections which secured warranted cold work, high accuracy, low roughness, but they did not lead to such values for workpieces with actual surface profiles. This first of all concerned special alloys. It was stated that one of the quality measures for surface layer determining operating properties of products (in particular endurance strength) is degree of cold work in high-load spots of workpieces. For example more than $0,5-1 \%$ alteration in degree of cold work in steels to any side from optimum value significantly lowers the efficiency of mechanical cold work obtained at recommended modes. You need to obtain properties of surface layer with stable specifications for correction of surface instability from cold work in previous processing. This is possible if you fulfill surface preparation by one of noncontact methods suitable for application in combined process. In this case design of combined process requires development of impact mechanisms in all contributory factors and their cooperation in treatment process.

Anodic removal of cold work layer from the surface provides conditions for regulation of initial data by the process, ensures consistent final outcome and lowers forces operating during mechanical action, especially at the final stage of surface layer formation.

The proposed technique of combined processing control allows management of stock alteration with the help of localized anodic dissolution and change of time needed for combined processing with initial stock removal of initial stock, including non-uniform one.

The completed investigations of channels processing showed that combination of anodic dissolution of metal and mechanical hardening according to the pattern of mandrelling ensures decrease of imprecision in comparisson with traditional mandrelling up to 5-6 quality grade with surface roughnesses previously obtained by liquid honing and fine polishing.

\section{Conclusions}

Gathered experience showed that outcome of combined processing in channels with direct cross-section may be fulfilled for holes with variable shape and cross-section area, also in treatment of outer surfaces made of any conductive materials of unspecified surface shape.

Implementation of the proposed technique according to proposed processing pattern is possible due to control of processing modes and use of feedback during shaping process.

Restrictions in force of mechanical effect of combined processing enables alteration in process parameters in relation to implementor's demands. If previously implementor was limited in technological capabilities mostly by mechanical processing, now combined processing techniques provide accuracy and quality of surface layer and worldclass operational factors, this opens door for international expansion.

\section{References}

1. A.G. Suslov, Directions of further development of engineering technology, Directory. Engineering Journal, v. 1, pp. 3-6 (2010) 
2. V.P. Smolentsev, A.V. Kuzovkin, A.I. Boldyrev etc., Technology of electrical processing methods (Voronezh State Tecnical Univercity, Voronezh, 2001)

3. V.P. Smolentsev, A.I. Boldyrev, A.V. Kuzovkin etc., Combined processing methods (Voronezh State Tecnical Univercity, Voronezh, 1996)

4. V.V. Berdnik, Grinding by conductive circles with the application of an electric field (High School, Kiev, 1984)

5. B.P. Saushkin etc., Physicochemical methods of processing in the production of gas turbine engines (Bustard, Moscow, 2002)

6. A.G. Suslov, Quality management of products operating under extreme conditions under non-stationary influences (Mechanical Engineering, Moscow, 2004)

7. A.P. Babichev, P.D. Motrenko, D.V. Maksimov etc., The use of vibro-impact processing to improve the quality and performance of power components of high-tech products, Technological methods of improving the quality of products in engineering, Voronezh State Tecnical Univercity, Voronezh, pp. 43-52 (2010)

8. A.I. Boldyrev, Technological support of quality of a surface layer at the combined processing in electrolyte with a filler, Journal of Orel State Tecnical Univercity, v. 3/275(560), pp. 63-66 (2009)

9. E.V. Smolentsev, Design of electrical and combined processing methods (Mechanical Engineering, Moscow, 2005)

10. A.I. Boldyrev, V.P. Smolentsev, Method of electrochemical treatment (USSR Patent №1085734, 1984) 